\title{
A Shape-based Decomposition of the Yield Adjustment Term in the Arbitrage-Free Nelson and Siegel (AFNS) model of the yield curve. by
}

\author{
James M. Steeley
}

\begin{abstract}
The appealing feature of the arbitrage-free Nelson-Siegel (AFNS) model of the yield curve is the ability to capture movements in the yield curve through readily interpretable shifts in its level, slope, or curvature, all within a dynamic arbitrage-free framework. To ensure that the level, slope and curvature factors evolve so as not to admit arbitrage, the model introduces a yield adjustment term. This paper shows how the yield adjustment term can also be decomposed into the familiar level, slope and curvature elements plus some additional readily interpretable shape adjustments. This means that, even in an arbitrage-free setting, it continues to be possible to interpret movements in the yield curve in terms of level, slope and curvature influences.
\end{abstract}

\section{JEL: G12}

Keywords: Arbitrage-free, Nelson-Siegel model, yield curve

\section{February 2014}

The author is grateful to Glenn Rudebusch, Francis Diebold and an anonymous Applied Financial Economics reviewer for helpful comments on an earlier version of the paper.

Address for correspondence: Prof. James M. Steeley, Aston Business School, Aston University, Birmingham, B4 7ET, UK. Tel: 0121-204-3248. Email: j.m.steeley@aston.ac.uk. 


\title{
A Shape-based Decomposition of the Yield Adjustment Term in the Arbitrage-Free Nelson and Siegel (AFNS) model of the yield curve.
}

\begin{abstract}
The appealing feature of the arbitrage-free Nelson-Siegel (AFNS) model of the yield curve is the ability to capture movements in the yield curve through readily interpretable shifts in its level, slope, or curvature, all within a dynamic arbitrage-free framework. To ensure that the level, slope and curvature factors evolve so as not to admit arbitrage, the model introduces a yield adjustment term. This paper shows how the yield adjustment term can also be decomposed into the familiar level, slope and curvature elements plus some additional readily interpretable shape adjustments. This means that, even in an arbitrage-free setting, it continues to be possible to interpret movements in the yield curve in terms of level, slope and curvature influences.
\end{abstract}

\section{JEL: G12}

Keywords: Arbitrage-free, Nelson-Siegel model, yield curve

February 2014 


\section{Introduction}

Since the pioneering work of McCulloch $(1971,1975)$, a huge literature has grown up devoted to both innovating and comparing alternative specifications to estimate the cross section of zerocoupon bond yields from the market prices of coupon-paying bonds. ${ }^{1}$ Among these techniques, the Nelson and Siegel (1987) parameterization has become widely adopted, by both financial institutions and central monetary authorities.

More recently, Diebold and Li (2006) developed a dynamic version of the Nelson and Siegel model by permitting the parameters to follow simple autoregressive processes. They pointed out that as the components of the Nelson and Siegel functional form can be interpreted as separate level, slope and curvature functions, they could be regarded as factor loadings resembling the level, slope and curvature factors arising from principal components decompositions of yield curve movements. They showed that the dynamic model could be estimated with ease, and had superior forecasting performance against a range of common alternatives.

Theoretical work, by Björk and Christensen (1999) and, more recently, by Christensen, Diebold and Rudebusch $(2009,2011)$ has shown that neither the Dynamic Nelson and Siegel model (DNS) nor its extension, by the addition of another curvature factor (the Svensson (1995) model), will be free from arbitrage. However, Christensen, Diebold and Rudebusch (2011) identify the necessary restrictions within the canonical three factor affine term structure model developed by Duffie and Kan (1996), to embed the Nelson and Siegel "shape factors". ${ }^{2}$ This delivers an

\footnotetext{
${ }^{1}$ Comparative studies and surveys include Anderson (1994), Deacon and Derry (1994), Bliss (1997), Ferguson and Raymar (1998), Anderson and Sleath (2001), Ioannides (2003) and Steeley (2008).

${ }^{2}$ Krippner (2010) has shown that models in the Nelson and Siegel class can arise as a formal Taylor approximation to the dynamic component of the generic Gaussian affine term structure model of Dai and Singleton (2002).
} 
arbitrage-free dynamic Nelson and Siegel (AFNS) model of the yield curve, which they show delivers further improvements in yield curve forecasting performance.

However, the AFNS model differs from the dynamic Nelson and Siegel model by the addition of a complicated function of maturity. Although Christensen, Diebold and Rudebusch (2011) show that this yield adjustment term introduces more flexibility into the shape of the yield curve, particularly at the long end of the curve, it does not appear to have visually interpretable characteristics like the level, slope and curvature components of the DNS model.

In this paper, I show how the yield adjustment term can be reconstituted into a set of components that contribute readily interpretable influences on the shape of the yield curve, including adjustments to each of the level, slope and curvature factors contained within the AFNS model. A shape-based decomposition of the yield curves in Christensen, Diebold and Rudebusch (2011) is empirically identified, using the estimates of the parameters from their AFNS models. These shape-based components of the yield adjustment term are seen to help to reveal why and how the AFNS model introduces greater flexibility into the shape of the yield curve than is permitted within the original Nelson and Siegel model and how the AFNS model is related to other models of the cross section of yields.

The remainder of the paper is structure as follows. Section 2 describes the Nelson and Siegel model and both the dynamic and arbitrage-free extensions. In Section 3, the shape-based decomposition of the yield adjustment term is developed and interpreted. In Section 4, the decomposition is applied to the yield curve models in Christensen, Diebold and Rudebusch (2011). Section 5 concludes. 


\section{The Arbitrage-Free Nelson and Siegel (AFNS) Model}

The Nelson and Siegel (1987) model assumes that the zero-coupon yield (spot interest rate), $y(\tau)$, for maturity $\tau$, can be represented by

$$
y(\tau)=\beta_{0}+\beta_{1}\left(\frac{1-e^{-\lambda \tau}}{\lambda \tau}\right)+\beta_{2}\left(\frac{1-e^{-\lambda \tau}}{\lambda \tau}-e^{-\lambda \tau}\right)
$$

where $\beta_{0}, \beta_{1}, \beta_{2}$ and $\lambda$ are the parameters to estimate. The corresponding instantaneous forward curve, related through $f(\tau)=y(\tau)+y^{\prime}(\tau) \tau$, is

$$
f(\tau)=\beta_{0}+\beta_{1} e^{-\lambda \tau}+\beta_{2} \lambda \tau e^{-\lambda \tau}
$$

The functional form of both the forward rate and the spot rate curves comprise a constant term and a combination of exponential decay functions and Laguerre functions (a polynomial times an exponential decay). These functional forms were chosen because they produce three components to the yield curve (and forward curve) that can be readily interpreted as representing level, slope and curvature. This can be seen in Figure 1 that plots the component functions of each of the yield curve and forward curve as a function of maturity.

The non-linear parameter, $\lambda$, controls the rate of exponential decay. Relatively large values of $\lambda$ generate a fast rate of decay enabling the model to provide relatively better fitting of shorter maturity yields, while relatively small values provide a slower rate of decay to give relatively better fitting at longer maturity yields. The parameter also controls the maturity at which the curvature 
components reach their maximum. ${ }^{3}$ The example in Figure 1 uses a range of maturities up to 120 months $(\tau=1,2, \ldots, 120)$ and sets the non-linear parameter $\lambda=0.06$.

Diebold and $\mathrm{Li}$ (2006) observed that if the parameters in equation (1) are permitted to time vary, then the exponential functions can be interpreted as factor loadings resembling the level, slope and curvature factors arising from principal components decompositions of yield curve movements. ${ }^{4}$ Thus the dynamic Nelson and Siegel (DNS) model is

$$
y_{t}(\tau)=L_{t}+S_{t}\left(\frac{1-e^{-\lambda \tau}}{\lambda \tau}\right)+C_{t}\left(\frac{1-e^{-\lambda \tau}}{\lambda \tau}-e^{-\lambda \tau}\right)
$$

where $L_{t}, S_{t}$ and $C_{t}$ are the level, slope and curvature factors.

In combination, the three shape-based components determine the overall shapes of yield curves that can be modelled by the Nelson-Siegel function form. One striking property of the functional form is that as maturity increases so both the spot and forward rates approach the value of the parameter $\beta_{0}$, if a single cross section is being considered, and approach the value of the level factor in a dynamic setting. This makes the Nelson-Siegel and its dynamic counterpart potentially inflexible models of longer term yields relative to, for example, spline-based models of the cross section of yields. ${ }^{5}$ Serendipitously, efforts to ensure no-arbitrage in the dynamic NelsonSiegel model, have introduced into the model some additional flexibility in modelling longer term yields.

\footnotetext{
${ }^{3}$ The forward rate curvature component reaches a maximum when $\tau=\frac{1}{\lambda}$, while the spot rate curvature component reaches a maximum when $e^{-\lambda \tau}\left(\lambda^{2} \tau^{2}+\lambda \tau+1\right)=1$.

${ }^{4}$ See, for example, Litterman and Scheinkman (1991) and Steeley (1990).

${ }^{5}$ See Steeley (2008) for a comparison of the relatively abilities of Nelson-Siegel and spline-based models to fit to the cross section of yields.
} 
Christensen, Diebold and Rudebusch (2011), hereafter CDR (2011), show that if (i) the three shape factors in equation (3) follow Gaussian Ornstein-Uhlenbeck processes with a constant volatility matrix, which they denoted by $\Sigma$, (ii) the first ("level”) factor is a unit root process under the risk neutral measure, (iii) the instantaneous short interest rate is the sum of the first and second ("slope") factors, (iv) the rate of mean reversion of the slope and curvature factor are both equal to $\lambda$, and (v) $\lambda$ is the scale by which deviations of the third (curvature) factor from its mean affect the mean of the second (slope) factor, then, under the risk neutral measure and in the absence of arbitrage, bond yields will be given by ${ }^{6}$

$$
y_{t}(\tau)=L_{t}+S_{t}\left(\frac{1-e^{-\lambda \tau}}{\lambda \tau}\right)+C_{t}\left(\frac{1-e^{-\lambda \tau}}{\lambda \tau}-e^{-\lambda \tau}\right)-\frac{A(\tau)}{\tau}
$$

The only difference with the DNS model, in equation (3), is the yield-adjustment term, $A(\tau)$, which depends on maturity, and the drifts and the volatility matrix of the three factors. ${ }^{7}$ By assuming that the drifts are zero under the risk neutral measure ${ }^{8}$ CDR (2011) show that the yield adjustment term is the following function of maturity and the elements of the volatility matrix

\footnotetext{
${ }^{6} \mathrm{CDR}$ (2011) formally derive the general restrictions on the affine class of arbitrage free models from which these specific restrictions and properties follow.

${ }^{7}$ The functional form in equation (4) arises as the solution to the ordinary differential equations that arise when the assumed risk-neutral dynamics of the three factors described earlier are combined with the relationship between bond prices and yields.

${ }^{8}$ This assumption is a necessary identifying restriction as the latent state variables (shape factors) may be arbitrarily rotated, see Singleton (2006). One of the advantages of the Nelson-Siegel class of models over the use of factor analysis is that the unit loading on the first factor is imposed from the outset potentially enhancing the efficiency of the estimates of the remaining factors, see CDR (2011).
} 


$$
\begin{aligned}
\frac{A(\tau)}{\tau} & =\bar{A}\left[\frac{\tau^{2}}{6}\right] \\
& +\bar{B}\left[\frac{1}{2 \lambda^{2}}-\frac{1}{\lambda^{3}} \frac{1-e^{-\lambda \tau}}{\tau}+\frac{1}{4 \lambda^{3}} \frac{1-e^{-2 \lambda \tau}}{\tau}\right] \\
& +\bar{C}\left[\frac{1}{2 \lambda^{2}}+\frac{1}{\lambda^{2}} e^{-\lambda \tau}-\frac{1}{4 \lambda} \tau e^{-2 \lambda \tau}-\frac{3}{4 \lambda^{2}} e^{-2 \lambda \tau}-\frac{2}{\lambda^{3}} \frac{1-e^{-\lambda \tau}}{\tau}+\frac{5}{8 \lambda^{3}} \frac{1-e^{-2 \lambda \tau}}{\tau}\right] \\
& +\bar{D}\left[\frac{1}{2 \lambda} \tau+\frac{1}{\lambda^{2}} e^{-\lambda \tau}-\frac{1}{\lambda^{3}} \frac{1-e^{-\lambda \tau}}{\tau}\right] \\
& +\bar{E}\left[\frac{3}{\lambda^{2}} e^{-\lambda \tau}+\frac{1}{2 \lambda} \tau+\frac{1}{\lambda} \tau e^{-\lambda \tau}-\frac{3}{\lambda^{3}} \frac{1-e^{-\lambda \tau}}{\tau}\right] \\
& +\bar{F}\left[\frac{1}{\lambda^{2}}+\frac{1}{\lambda^{2}} e^{-\lambda \tau}-\frac{1}{2 \lambda^{2}} e^{-2 \lambda \tau}-\frac{3}{\lambda^{3}} \frac{1-e^{-\lambda \tau}}{\tau}+\frac{3}{4 \lambda^{3}} \frac{1-e^{-2 \lambda \tau}}{\tau}\right]
\end{aligned}
$$

where $\bar{A}=\sigma_{11}^{2}+\sigma_{12}^{2}+\sigma_{13}^{2}, \bar{B}=\sigma_{21}^{2}+\sigma_{22}^{2}+\sigma_{23}^{2}, \bar{C}=\sigma_{31}^{2}+\sigma_{32}^{2}+\sigma_{33}^{2}, \bar{D}=\sigma_{11} \sigma_{21}+$ $\sigma_{12} \sigma_{22}+\sigma_{13} \sigma_{23}, \bar{E}=\sigma_{11} \sigma_{31}+\sigma_{12} \sigma_{32}+\sigma_{13} \sigma_{33}, \bar{F}=\sigma_{21} \sigma_{31}+\sigma_{22} \sigma_{32}+\sigma_{23} \sigma_{33}$, are constants, and where $\sigma_{i j}$ are the components of the volatility matrix of the three factors ${ }^{9}$

$$
\Sigma=\left(\begin{array}{lll}
\sigma_{11} & \sigma_{12} & \sigma_{13} \\
\sigma_{21} & \sigma_{22} & \sigma_{23} \\
\sigma_{31} & \sigma_{32} & \sigma_{33}
\end{array}\right)
$$

CDR (2011, Figure 1) plot the function (5), as one whole entity, using parameters estimated from monthly unsmoothed Fama-Bliss (1987) zero-coupon yields from January 1987 to December 2002. ${ }^{10}$ Where the three factors are independent, they find that the yield adjustment term is monotonically downwards concave, but where the three factors are correlated, the yield adjustment term takes a generally downwards slope but with a distinct upward inflexion between maturities of 10 and 20 years. To understand why the yield adjustment term takes these forms, and to enable

\footnotetext{
${ }^{9}$ See CDR (2011, equation (3)) for further details. This decomposition arises from solving the ordinary differential equation that defines the yield adjustment term in terms of the underlying parameters of the dynamic factors.

${ }^{10}$ The parameter estimates are in CDR (2011) Tables 5 and 6.
} 
greater insight into how the shape of the yield curve is being affected, a new decomposition of the yield adjustment term is developed in this paper. This decomposition recombines the six elements of equation (5) in a manner that permits greater insight into the role of the yield adjustment term.

\section{A Shape-based Decomposition of the Yield Adjustment Term}

Like the shape-based loadings on the dynamic factors in the AFNS model in equation (4), the yield adjustment term as presented in equation (5) also contains exponential decays and products of polynomials and exponential decays. These elements of the yield adjustment term will, therefore, contain familiar shape-based characteristics. The following decomposition of the yield adjustment term collects together these familiar elements and separates them from other elements, which are also able to be given shape-based interpretations. Instead of being comprised the six elements, in equation (5), the yield adjustment term, can instead be represented as the sum of four distinct shape-based components, as follows:

$$
\frac{A(\tau)}{\tau}=A N S(\tau)+C D R 5(\tau)+F N S(\tau)+Q F(\tau)
$$

where

$$
\begin{aligned}
& A N S(\tau)=\left[\frac{\bar{B}+\bar{C}+2 \bar{F}}{2 \lambda^{2}}\right]-\left[\frac{\bar{B}+\bar{C}+2 \bar{F}}{\lambda^{2}}\right]\left(\frac{1-e^{-\lambda \tau}}{\lambda \tau}\right)-\left[\frac{\bar{C}+\bar{D}+3 \bar{E}+\bar{F}}{\lambda^{2}}\right]\left(\frac{1-e^{-\lambda \tau}}{\lambda \tau}-e^{-\lambda \tau}\right) \\
& \operatorname{CDR5}(\tau)=\left[\frac{2 \bar{B}+2 \bar{C}+4 \bar{F}}{\lambda_{2}^{2}}\right]\left(\frac{1-e^{-\lambda_{2} \tau}}{\lambda_{2} \tau}\right)+\left[\frac{3 \bar{C}+2 \bar{F}}{\lambda_{2}^{2}}\right]\left(\frac{1-e^{-\lambda_{2} \tau}}{\lambda_{2} \tau}-e^{-\lambda_{2} \tau}\right) \\
& F N S(\tau)=\bar{E}\left(\frac{\tau e^{-\lambda \tau}}{\lambda}\right)-\left[\frac{\bar{C}}{2}\right]\left(\frac{\tau e^{-\lambda_{2} \tau}}{\lambda_{2}}\right)
\end{aligned}
$$


$Q F(\tau)=\left[\frac{\bar{D}+\bar{E}}{2 \lambda}\right] \tau+\left[\frac{\bar{A}}{6}\right] \tau^{2}$

where the substitution $\lambda_{2}=2 \lambda$ has also been made. With this alternative representation of the yield adjustment term it is possible to see where and how the increased yield curve flexibility of the AFNS model arises.

The first component of the re-arranged yield adjustment term, equation (8), comprises three functions providing a direct adjustment to each of the level, slope and curvature loadings in the AFNS model. The level of the curve has is being adjusted by $\left[\frac{\bar{B}+\bar{C}+2 \bar{F}}{2 \lambda^{2}}\right]$, the slope by $\left[\frac{\bar{B}+\bar{C}+2 \bar{F}}{\lambda^{2}}\right]$ and the curvature by $\left[\frac{\bar{C}+\bar{D}+3 \bar{E}+\bar{F}}{\lambda^{2}}\right]$. We call this component of the yield adjustment term the "adjusted NelsonSiegel" component and label this $\operatorname{ANS}(\tau)$.

The second component, equation (9), comprises an additional slope term, $\left[\frac{2 \bar{B}+2 \bar{C}+4 \bar{F}}{\lambda_{2}^{2}}\right]\left(\frac{1-e^{-\lambda_{2} \tau}}{\lambda_{2} \tau}\right)$, and an additional curvature term, $\left[\frac{3 \bar{C}+2 \bar{F}}{\lambda_{2}^{2}}\right]\left(\frac{1-e^{-\lambda_{2} \tau}}{\lambda_{2} \tau}-e^{-\lambda_{2} \tau}\right)$, where the non-linear parameter, $\lambda_{2}$, is double its value in the loadings on the Nelson and Siegel slope and curvature factors. Svensson (1995) was the first to recommend adding an additional curvature term to the original Nelson and Siegel formulation to enhance the flexibility of the fit, while Björk and Christensen (1999) proved that an additional slope term (with a doubled non-linear parameter) would be consistent with a certain class of no-arbitrage models. The shaped based decomposition of the yield adjustment term, shows that both of these earlier extensions of the Nelson and Siegel model are represented in the AFNS model. In fact, in related work, Christensen, Diebold and Rudebusch (2009), hereafter CDR (2009), have combined the benefits of both of these developments into their five factor extended version of the AFNS model. Their five factor model has both an extra slope factor and an extra 
curvature factor, but does not need the restriction that $\lambda_{2}=2 \lambda$. Thus, the adjusted yield term in the (three factor) AFNS model is actually building in some of the additional flexibility of the CDR (2009) extended five factor model, but by restricting $\lambda_{2}=2 \lambda$ (as in the four factor Björk and Christensen (1999) model) is doing so in a manner that ensures no-arbitrage in the three factor setting. This component of the yield adjustment term we label the $C D R 5(\tau)$ component.

The component of the yield adjustment term in equation (10) comprises two additional curvature terms, but they are not in the form found in the yield curve specifications of Nelson and Siegel models. Instead, these terms have the functional form of the curvature terms in Nelson and Siegel forward curves. Specifically, they are the product of a polynomial in maturity and an exponential decay, see equation (2). Although the non-linear parameters, $\lambda$ and $\lambda_{2}$, are in the denominator in equation (10) whereas they are in the numerator in equation (2), it is the functional form of the Laguerre function (the product of a polynomial and an exponential decay) that drives the shape of these curvature functions (see Nelson and Siegel (1987, Figure 2)). Moreover, as the forward curve is related to the rate of change (along the maturity range) of the spot rate, these terms can be regarded as representing a change in curvature along the spot curve. These components of shape would be consistent therefore with the eignvector of a fourth (orthogonal) principal component, in a principal components analysis of the covariance matrix of yields, and are capturing a further dimension of yield curve shape. We call these two shape functions the Forward curvature Nelson-Siegel adjustment and label them as $F N S(\tau)$. From the definitions of the relation between the constants and the components of the volatility matrix, equation (6), it can be seen that the term involving $\lambda$ will not feature in the model if the shape factors are independent.

The final component, equation (11), comprises two elements of a quadratic function of maturity, labelled $Q F(\tau)$. The appearance of a term in the square of maturity as a component of the 
yield adjustment term is not unexpected in this AFNS model, because the first ("level") factor is a constrained to be a random walk under the risk neutral measure. In a simple one-factor Gaussian random walk model of the short interest rate, Brown and Schaefer (1994) have shown that the nonlinear relationship between bond prices and yields generates a Jensen's inequality term that is equal to one half of the variance of the short rate times the square of maturity. In this final component of the yield adjustment term in the three factor AFNS model, there is also a term in the square of maturity. From equation (11), it can be seen that this square of maturity term is scaled only by the parameter $\bar{A}$, which depends on the square of the volatility term relating only to the first factor. ${ }^{11}$ In the AFNS model, the first factor is assumed to be a random walk process. Therefore, this term in the shape-based decomposition would seem to be picking up this same Jensen's inequality effect, which arises in a simple one-factor model, but in the more general AFNS model. Brown and Schaefer (1994) have shown that a Jensen's inequality term can dominate the shape of the yield curve at the long end, but that this can both improve the fit of yield curves and measures of interest rate expectations. It is likely, therefore, that the term in the square of maturity in the yield adjustment term is a key contributor to the improvements to yield curve shape flexibility and fit in the AFNS model, relative to the DNS model, found by CDR (2011). The other term in equation (11) is a linear term in maturity, which only influences the curve when the shape factors are correlated. It too has precedent in other arbitrage-free models. Björk and Christensen (1999) augment a dynamic Nelson and Siegel model with both an extra slope factor and a linear function of maturity. ${ }^{12}$

\footnotetext{
${ }^{11}$ Strictly it is only the square of the volatility of the first factor when the three shape factor are independent, but identification of the AFNS model parameters requires the covariance components of $\bar{A}$ to be set of zero, see CDR (2011) and Table 1.

${ }^{12}$ Earlier still, Chambers, Carleton and Waldman (1984) proposed an exponential polynomial model to estimate the discount function, which also implies a polynomial function for the yield curve.
} 


\section{Identifying the Shape-based Decomposition for the CDR (2011) yield curves}

To see how the yield adjustment term can be decomposed into meaningful shape-based components, we calibrate the individual components of this new decomposition, equations (7) to (11), using the parameter estimates used to model the yield curves in CDR (2011, Tables 5 and 6).

In Figures 2 and 3, the overall yield adjustment term, in CDR (2011, Figure 1), is disaggregated into the individual components identified in the new decomposition in equations (7) through (11). We use the parameter estimates in CDR (2011, Tables 5 and 6) to identify the constants in equations (7) through (11), that is, $\bar{A}, \bar{B}, \bar{C}, \bar{D}, \bar{E}$ and $\bar{F}$. These constants and the CDR estimates are given in Table 1. We then use these constants in equations (7) through (11) to generate Figures 2 and 3, which show the shaped-based components of the yield adjustment term, as a function of maturity.

It can be seen that the values of $\bar{A}$ and $\bar{C}$ are an order of magnitude different between the independent factors case and the correlated factors case, while the value of $\bar{B}$ remains broadly unchanged. As these constants are calculated from the estimated values of the parameters of the volatility matrix, the differences are coming from both the differences in the estimated parameters of this matrix and the differing model specification. In the independent case, only the diagonal elements of this matrix are estimated, while the off-diagonal elements are set to zero. In the correlated case, the lower triangular elements of the matrix are estimated. Thus, the correlated case has three additional degrees of freedom that the yield adjustment term can use to fit to the shape of the curve. The estimated parameters may therefore differ substantially when a model with more degrees of freedom is optimized to fit the yield curve's shape. A key message of this paper is that, 
however, it is not the parameters per se that give the new insights, but how they combine not only within the constants $\bar{A}, \bar{B}, \bar{C}, \bar{D}, \bar{E}$ and $\bar{F}$, but also within the specific shape-based components of the yield adjustment term identified in equations (7) to (11). We now turn to this specific question.

In Figure 2, the three factors (level, slope and curvature) are restricted to be independent. In this case, three of the constants in the yield adjustment term, $\bar{D}, \bar{E}$ and $\bar{F}$, will all take the value zero, and the AFNS yield adjustment term in cross section comprises an adjustment to the standard Nelson Siegel model (equation (8)), with an extra slope and an extra curvature term (equation (9)), a term capturing changes in the curvature of the second curvature term (one element of equation (10), and a term in the square of maturity (one element of equation (11). From Figure 2, it can be seen clearly that the dominant component of the yield adjustment term for the CDR (2011) yield curves is the square of maturity. While CDR (2011, footnote 19) observe that the difference between the DNS model and the AFNS model is that the yield curve must in the absence of arbitrage (by Jensen's inequality) slope downwards at the long end, the direct link to the quadratic term within in the yield adjustment term is not made. Figure 2 shows that, for the CDR (2011) dataset, this is what is likely to be driving the yield adjustment term and generating the difference in fit between the DNS and AFNS models.

The possible dominance of the square of maturity in determining the yield adjustment term is not however just an empirical artefact, but is actually built into the deeper structure of the yield adjustment term. This only becomes apparent within the decomposition in equations (7) to (11). In the $\operatorname{ANS}(\tau)$ and the $\operatorname{CDR} 5(\tau)$ constituents of the decomposition, equations (8) and (9), it can be seen that the two slope terms have opposite signs, as do the two curvature terms. This means that there will likely be some off-setting influences of the $C D R 5(\tau)$ components upon the $\operatorname{ANS}(\tau)$ 
components, permitting the other constituents of the decomposition, in particular the quadratic function, to exert more influence on the shape of the curve.

In Figure 3, the level, slope and curvature factors are not restricted to be independent, and the yield adjustment term displays a hump shape (inflexion), where the adjustment term declines at maturities up to 10 years, then rises again, before falling sharply from 20 years to maturity onwards. The decomposition of the yield adjustment term provided by equations (7) to (11) and depicted in Figure 3 makes clear what is driving this for the CDR (2011) dataset. As the estimates of the constants, $\bar{D}$ and $\bar{E}$ are both negative, the impact of the linear term in maturity is overall positive, see equations (5) and (11). When combined with the other components, this more than offsets the downwards adjustment due to the quadratic term between maturities of between 10 and 20 years, but beyond 20 years the quadratic term dominates once again. At maturities of up to 10 years, the adjustment is still heavily influenced by the quadratic term, although other factors also have some influence too in this range.

In Figure 2, it can also be seen that the change in curvature factor, from equation (10), has an almost negligible effect on the shape of the yield adjustment term. The same is the case for the

correlated-factor AFNS model where, for clarity in Figure 3, these are not drawn in. This small impact of the change in slope and curvature terms is consistent with the tiny influence of fourth and higher principal components of the yield curve, as observed by, for example, Steeley (1990).

\section{Summary and Conclusion}


A shape-based decomposition of the yield adjustment term in the arbitrage-free Nelson-Siegel (AFNS) model of CDR (2011) is developed. This enables the yield adjustment term to be separated into the direct effects on the familiar shape characteristics (level, slope and curvature) of the underlying dynamic Nelson-Siegel model, and other shape-based effects. The latter includes a quadratic function of maturity, arising as a consequence of Jensen's inequality that may dominate the shape of the yield adjustment term and is likely to be the key contributor to the improvements in fit offered by the AFNS model of CDR (2011). The new decomposition restores the ability to fully interpret the movements of the yield curve in terms of level, slope and curvature within an arbitrage-free setting.

\section{References}

Anderson, N., 1994. Testing models of the yield curve. Bank of England mimeo.

Anderson, N., Sleath, J., 2001. New estimates of the UK real and nominal yield curves. Bank of England, working paper.

Björk, T., Christensen, B., 1999. Interest rate dynamics and consistent forward curves. Mathematical Finance 9, 323-348

Bliss, R.R., 1997. Testing term structure estimation methods. Advances in Futures and Options Research, 9, 197-231. 
Brown, R.H., Schaefer, S.M., 1994. Interest rate volatility and the shape of the term structure. Philosophical Transactions of the Royal Society 347, 563-576.

Chambers, D.R., Carleton, W.T., Waldman, D.W., 1984. A new approach to estimation of the term structure of interest rates. Journal of Financial and Quantitative Analysis 19, 233-52.

Christensen, J.H.E., Diebold, F.X., and Rudebusch, G.D., 2009. An arbitrage-free generalized Nelson-Siegel term structure model. Econometrics Journal 12, C33-C64.

Christensen, J.H.E., Diebold, F.X., and Rudebusch, G.D., 2011. The affine arbitrage-free class of Nelson-Siegel term structure models. Journal of Econometrics 164, 4-20.

Dai, Q., and K. Singleton, 2002, Expectation puzzles, time varying risk premia and affine models of the term structure, Journal of Financial Economics 63, 415-441.

Deacon, M., Derry, A.,1994. Estimating the term structure of interest rates. Bank of England working paper series, no. 23 .

Diebold, F.X., Li, C., 2006. Forecasting the term structure of government bond yields. Journal of Econometrics 130, 337-364.

Diebold, F.X., Rudebusch, G.D., 2013. The Dynamic Nelson-Siegel Approach to Yield Curve Modelling and Forecasting (Princeton University Press, Princeton, NJ).

Duffie, D., Kan, R., 1996. A yield-factor model of interest rates. Mathematical Finance 6, 379-406.

Fama, E.F., Bliss, R.R., 1987. The information in long-maturity forward rates. American Economic Review 77, 680-692. 
Ferguson, R., Raymar, S., 1998. A comparative analysis of several popular term structure estimation models. Journal of Fixed Income, March, 17-33.

Ioannides, M., 2003. A comparison of yield curve estimation techniques using UK data. Journal of Banking and Finance, 27, 1-26.

Krippner, L., 2010, A theoretical foundation for the Nelson and Siegel class of yield curve models, and an empirical application to U.S. yield curve dynamics, Federal Reserve Bank of New Zealand, Discussion paper no.2010/11.

Litterman, R., Scheinkman, J., 1991. Common factors affecting bond returns. Journal of Fixed Income $1,77-85$.

McCulloch, J.H., 1971. Measuring the term structure of interest rates. Journal of Business 44, 1931.

McCulloch, J.H., 1975. The tax adjusted yield curve. Journal of Finance 30, 811-830.

Nelson C.R., Siegel A.F., 1987. Parsimonious modelling of yield curves. Journal of Business 60, 473-489.

Singleton,K.J, 2006. Empirical Dyanamic Asset Pricing, Prinecton University Press, Princeton.

Steeley, J.M., 1990, Modelling the Dynamics of the Term Structure of Interest Rates, Economic and Social Review - Symposium on Finance 21, 337-362.

Steeley, J.M., 2008, Testing term structure estimation methods: Evidence from the UK STRIPS Market, Journal of Money, Credit and Banking 40, 1489-1512. 
Svensson L.E.O., 1995. Estimating forward interest rates with the extended Nelson-Siegel method. Sveriges Riksbank, Quarterly Review 3, 13-26. 
Figure 1: Level, slope and curvature components of Nelson and Siegel spot and forward curves

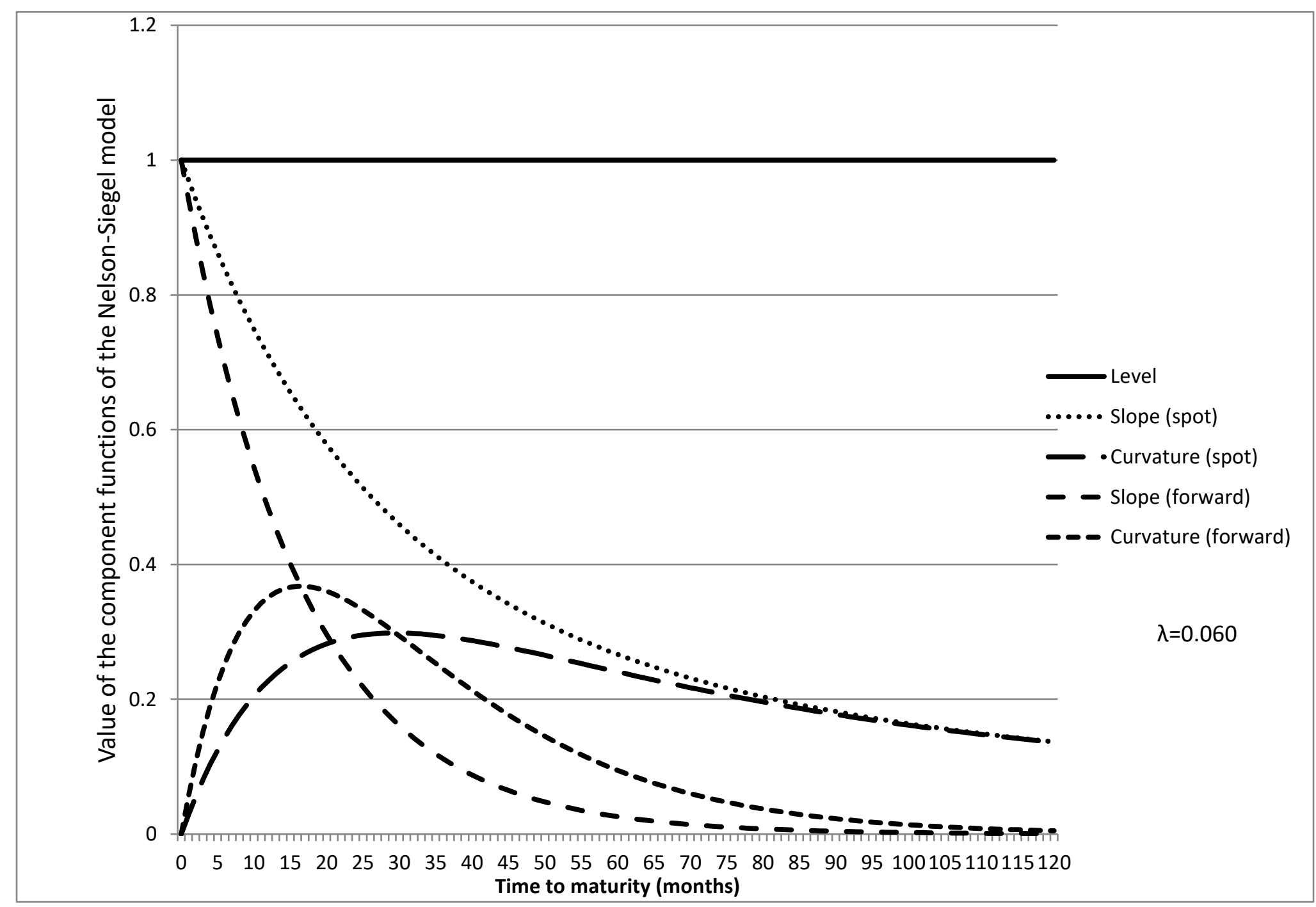


Figure 2: Components of the Yield Adjustment Term (independent factors)

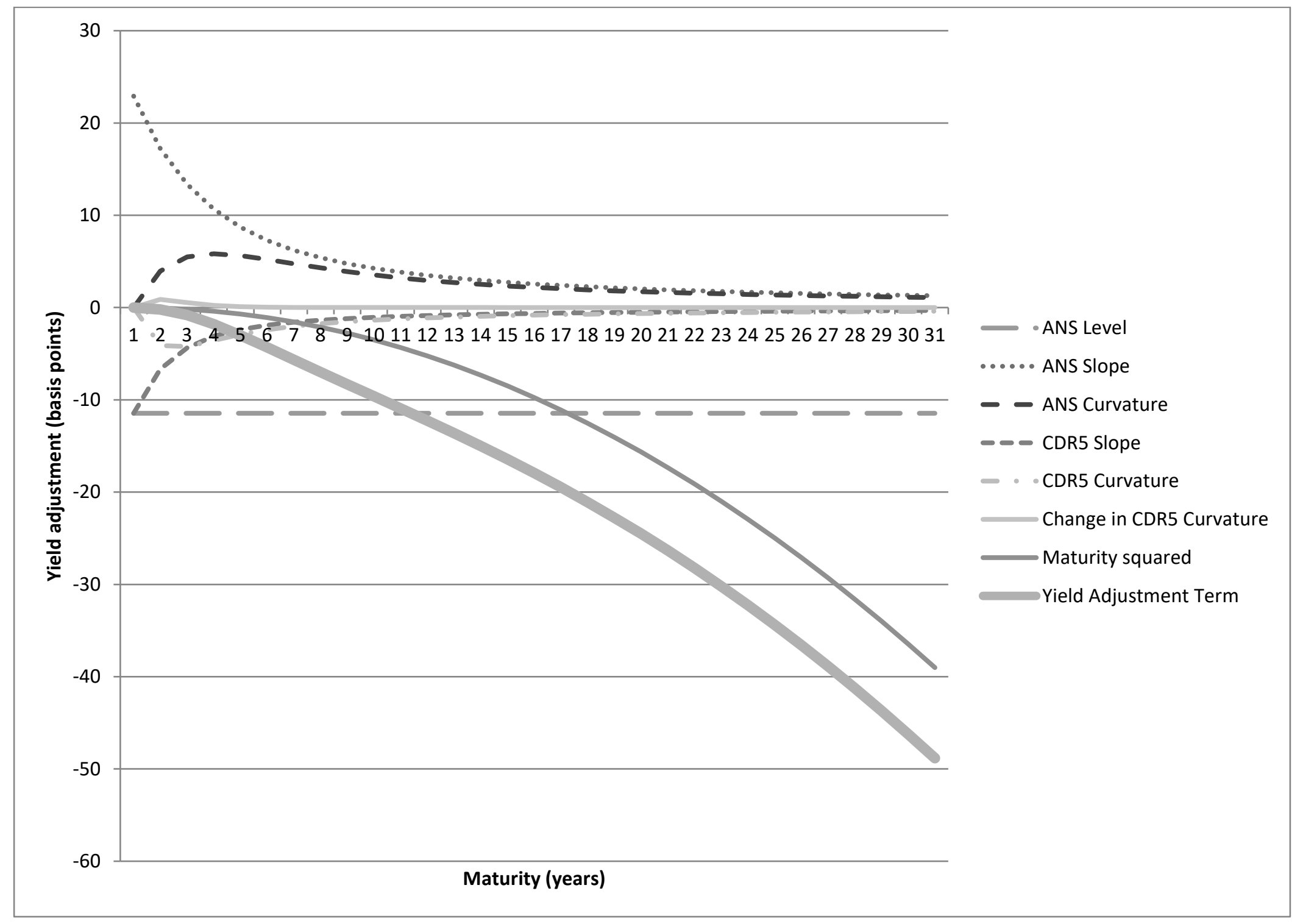


Figure 3: Components of the Yield Adjustment Term (correlated factors)

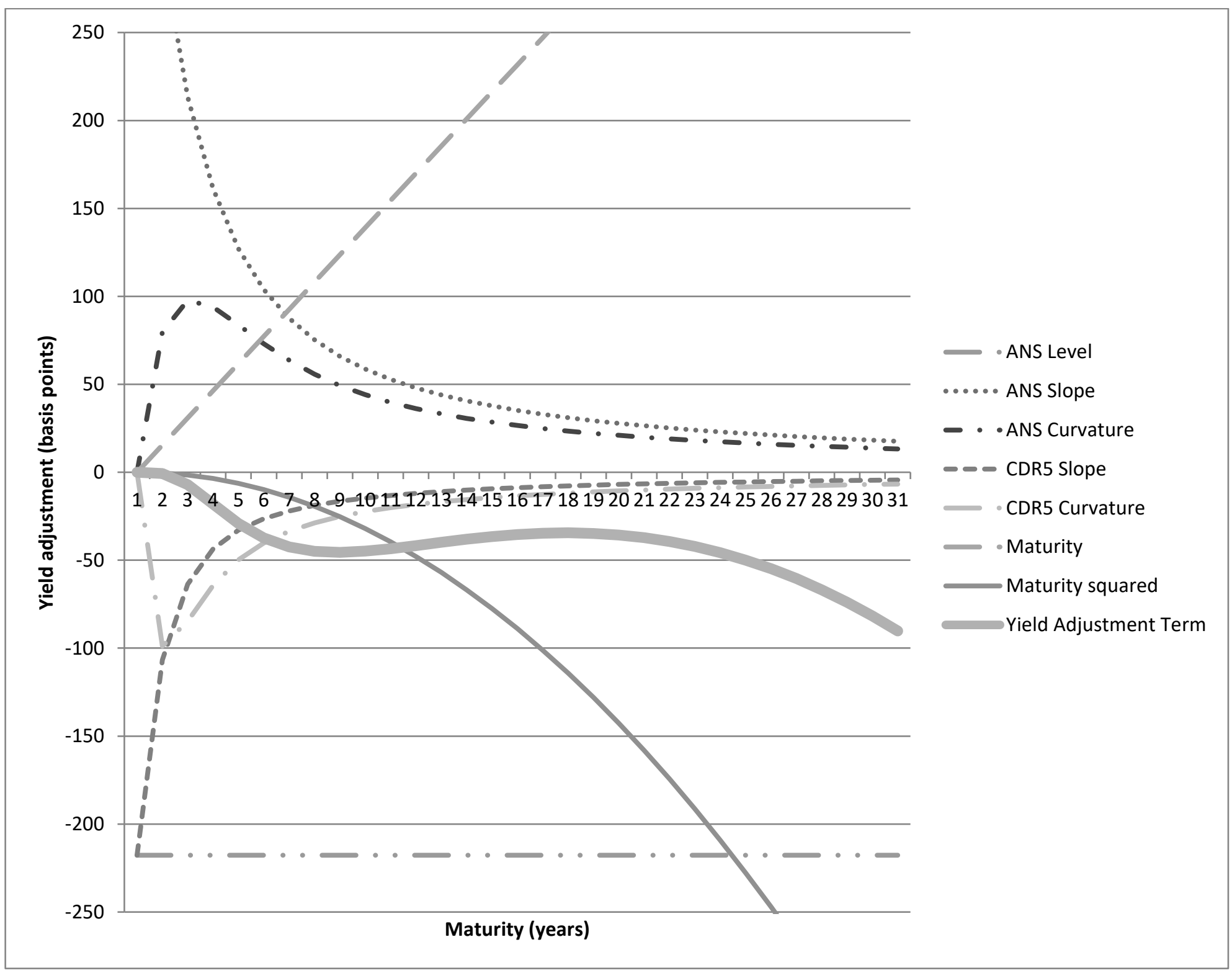


Table 1: Estimated parameters for the shape-based decomposition

\begin{tabular}{|c|c|c|c|c|c|c|c|c|c|c|c|c|c|}
\hline \multicolumn{14}{|c|}{$\begin{array}{l}\text { Estimates of the rate of decay, } \lambda \text {, in equation (4) and elements of the volatility matrix, } \Sigma \text {, are obtained from CDR (2011). CDR (2011) } \\
\text { show that the volatility matrix has to be triangular and so only the lower triangular elements of the matrix are estimated. The standard } \\
\text { errors of the estimated parameters are given in parentheses below the parameter values. These estimates are used to construct the } \\
\text { constants, } \bar{A} \text { to } \bar{F} \text {, (multiplied by } 10^{3} \text { ) that underpin the new decomposition of the yield adjustment term in equations (7) to (11). } \\
\text { These constants are calculated using the following formulae: } \bar{A}=\sigma_{11}^{2}+\sigma_{12}^{2}+\sigma_{13}^{2}, \bar{B}=\sigma_{21}^{2}+\sigma_{22}^{2}+\sigma_{23}^{2}, \bar{C}=\sigma_{31}^{2}+\sigma_{32}^{2}+\sigma_{33}^{2} \\
\bar{D}=\sigma_{11} \sigma_{21}+\sigma_{12} \sigma_{22}+\sigma_{13} \sigma_{23}, \bar{E}=\sigma_{11} \sigma_{31}+\sigma_{12} \sigma_{32}+\sigma_{13} \sigma_{33}, \bar{F}=\sigma_{21} \sigma_{31}+\sigma_{22} \sigma_{32}+\sigma_{23} \sigma_{33} \text {. The first row of parameter estimates } \\
\text { is for the AFNS model with independent factors, while the second row of parameter estimates is for the AFNS model with correlated } \\
\text { factors. }\end{array}$} \\
\hline Factors & $\lambda$ & $\sigma_{11}$ & $\sigma_{21}$ & $\sigma_{22}$ & $\sigma_{31}$ & $\sigma_{32}$ & $\sigma_{33}$ & $\overline{\bar{A}}$ & $\bar{B}$ & $\bar{C}$ & $\bar{D}$ & $\bar{E}$ & $\bar{F}$ \\
\hline Independent & $\begin{array}{l}0.5975 \\
(0.0015)\end{array}$ & $\begin{array}{l}0.0051 \\
(0.0001)\end{array}$ & & $\begin{array}{l}0.0110 \\
(0.0006)\end{array}$ & & & $\begin{array}{l}0.0264 \\
(0.0014)\end{array}$ & 0.0260 & 0.1210 & 0.6970 & & & \\
\hline Correlated & $\begin{array}{l}0.8244 \\
(0.0122)\end{array}$ & $\begin{array}{l}0.0154 \\
(0.0004)\end{array}$ & $\begin{array}{l}-0.0013 \\
(0.0051)\end{array}$ & $\begin{array}{l}0.0117 \\
(0.0018)\end{array}$ & $\begin{array}{l}-0.1641 \\
(0.0069)\end{array}$ & $\begin{array}{l}-0.0590 \\
(0.0106)\end{array}$ & $\begin{array}{l}0.0001 \\
(6.890)\end{array}$ & 0.2372 & 0.1386 & 30.409 & -0.0200 & -2.5271 & -0.4770 \\
\hline
\end{tabular}

